\title{
Hybrid FFT-ADALINE algorithm with fast estimation of harmonics in power system
}

\begin{abstract}
Hybrid fast Fourier transform Adaptive LINear Element (FFT-ADALINE) algorithm for fast and accurate estimation of harmonics is proposed in this study. The FFT method can perform fast conversion from time domain to frequency domain, but it cannot respond immediately to any change of the measured harmonics due to the utilisation of buffer. Meanwhile, ADALINE has better capability to respond immediately due to its learning ability, but its settling time is about two cycles of the measurement signal. In the proposed method, both of the aforementioned algorithms are combined for harmonic estimation where it is able to respond immediately to any change of the measured harmonics and the settling time is reduced to half cycle of the measurement signal. The theory of the proposed algorithm is the application of FFT with weights updating rule to reduce the error of ADALINE instantaneously. The robustness of the proposed method is simulated via MATLAB Simulink. The validity of the simulation work is further proven by the experimental work, which has been done with Chroma programmable AC source model 6590 and non-linear load operations. The proposed algorithm operates in good and accurate performance with the settling time is within half cycle.
\end{abstract}

Keyword: Hybrid FFT-ADALINE algorithm; Fast estimation; Harmonics; Power system 